\title{
Re: Safety of Dual Kidney Transplantation Compared to Single Kidney Transplantation from Expanded Criteria Donors: A Single Center Cohort Study of 39 Recipients
}

\author{
Mendel L1, Albano L2, Bentellis I1, Yandza T1, Bernardi C3, Quintens H1, Tibi B1, Jourdan J1, Durand M11, Amiel J1, Chevallier D1 \\ 1 University Hospital of Nice, Department of Urology, Nice, France \\ 2University Hospital of Nice, Department of Nephrology, Nice, France \\ 3 University Hospital of Nice, Department of Forensic Medicine, Nice, France \\ Transpl Int. 2018;31:1110-1124. doi: 10.1111/tri.13280. Epub 2018 Jun 5.
}

\section{EDITORIAL COMMENT}

Dual kidney transplantation (DKT) has been developed in order to compensate for nephron mass reduction and to use kidneys from very marginal donors that would have been discarded for single kidney transplantation (SKT). DKT is transplanting two kidneys from the same donor to a single recipient ipsilaterally or bilaterally. In this single center retrospective study, the authors compared the outcomes of DKT $(n=39)$ with that of SKT $(n=155)$ performed with grafts from expanded-criteria donors over an 8-year period and followed for a median of 36 and 26.5 months respectively, in recipients $\geq 65$ years of age, focusing on surgical outcomes. Although the authors observed a higher venous thrombosis rate in the DKT group (12.8\% vs 3.2\%) ( $p=0.02)$, similar graft survivals and better glomerular filtration rate over 24 months posttransplant were observed in DKT group $\left(45.0 \pm 16.3 \mathrm{vs} 39.8 \pm 13.8 \mathrm{~mL} / \mathrm{min} / 1.73 \mathrm{~m}^{2}\right.$; $\mathrm{p}=0.04$ ). DKT seems to remain a promising strategy to address the growing graft shortage in elderly patients with shorter waiting time on the waiting list, however, these data need to be confirmed by larger randomized controlled studies.

Yarkın Kamil Yakupoğlu, MD

๑Copyright 2018 by the Association of Urological Surgery / Journal of Urological Surgery published by Galenos Publishing House. 